\title{
Clock-Talk: Interactions between Central and Peripheral Circadian Oscillators in Mammals
}

\author{
Ueli Schibler,${ }^{1}$ Ivana Gotic, ${ }^{1}$ Camille Saini, ${ }^{1,2}$ Pascal Gos,${ }^{1}$ Thomas Curie,${ }^{3}$ \\ Yann Emmenegger, ${ }^{3}$ Flore Sinturel, ${ }^{1}$ Pauline Gosselin, ${ }^{1}$ Alan Gerber, ${ }^{1,4}$ \\ Fabienne Fleury-Olela, ${ }^{1}$ Gianpaolo Rando, ${ }^{1}$ Maud Demarque, ${ }^{1}$ and Paul Franken ${ }^{3}$ \\ ${ }^{1}$ Department of Molecular Biology, University of Geneva, CH-1211 Geneva, Switzerland \\ ${ }^{2}$ Laboratory of Circadian Endocrinology, Geneva University Hospitals, CH-1205 Geneva, Switzerland \\ ${ }^{3}$ Center of Integrative Genomics, University of Lausanne, CH-1015 Lausanne, Switzerland \\ ${ }^{4}$ Laboratory of Biochemistry and Molecular Biology, Rockefeller University, New York, New York 10065 \\ Correspondence: ueli.schibler@unige.ch
}

\begin{abstract}
In mammals, including humans, nearly all physiological processes are subject to daily oscillations that are governed by a circadian timing system with a complex hierarchical structure. The central pacemaker, residing in the suprachiasmatic nucleus (SCN) of the ventral hypothalamus, is synchronized daily by photic cues transmitted from the retina to SCN neurons via the retinohypothalamic tract. In turn, the SCN must establish phase coherence between self-sustained and cell-autonomous oscillators present in most peripheral cell types. The synchronization signals (Zeitgebers) can be controlled more or less directly by the SCN. In mice and rats, feeding-fasting rhythms, which are driven by the SCN through rest-activity cycles, are the most potent Zeitgebers for the circadian oscillators of peripheral organs. Signaling through the glucocorticoid receptor and the serum response factor also participate in the phase entrainment of peripheral clocks, and these two pathways are controlled by the SCN independently of feeding-fasting rhythms. Body temperature rhythms, governed by the SCN directly and indirectly through rest-activity cycles, are perhaps the most surprising cues for peripheral oscillators. Although the molecular makeup of circadian oscillators is nearly identical in all cells, these oscillators are used for different purposes in the SCN and in peripheral organs.
\end{abstract}

The rotation of the Earth around its own axis generates daily light-dark cycles that affect the lifestyle of all photosensitive organisms from cyanobacteria to humans. The phylogenetic adaptation to recurring daily environmental changes resulted in diurnal activity cycles, driven by time periods during which food availability is high, predator abundance low, and temperature, humidity, and/or lighting conditions compatible with an organism's lifestyle. Anticipating these changes - rather than just reacting to them-is expected to increase the fitness of organisms by optimizing their physiology and behavior with regard to both external factors and internal time-sensitive parameters. Indeed, most photosensitive organisms from photosynthetic bacteria to mammals have evolved internal timing devices, known as circadian clocks, which coordinate behavior and physiology in an anticipatory fashion. For example, plants induce the expression of photosynthetic genes a few hours before sunrise to optimize photosynthesis (Dodd et al. 2005), marine zooplankton species swim toward deeper layers at dawn or during the night before genotoxic ultraviolet light hits them (Gehring and Rosbash 2003; Tosches et al. 2014), and mice up-regulate the expression of hepatic detoxification enzymes before the activity period during which they absorb food and associated toxins (Gachon and Firsov 2011; Zmrzljak and Rozman
2012; DeBruyne et al. 2014). Obviously, the temporal regulation of physiology by circadian clocks works hand in hand with acute signaling pathways. This can be exemplified by glucose homeostasis. A sudden increase of blood glucose provoked by the consumption of carbohydrates leads to the acute secretion of insulin by pancreatic $\beta$ cells. In turn, insulin triggers multiple responses in peripheral tissues that lead to a decrease in blood sugar levels. Although this acute pathway is operative throughout the day, the efficiency of glucose-stimulated insulin secretion by $\beta$ cells and insulin sensitivity in target tissues oscillates in a circadian manner (Sadacca et al. 2011).

In addition to anticipating recurrent events inside and outside the organism, oscillators provide two other important benefits: (i) the temporal separation of chemically incompatible pathways and (ii) the limitation of potentially harmful, but necessary, chemical reactions to the times when they are required. To provide an example for the sequestration of incompatible processes, the circadian clock of most diazotrophic cyanobacteria sequesters nitrogen fixation to the night, so that oxygen produced during the day as a product of photosynthesis does not poison the nitrogenase activity (Mohr et al. 2013). To illustrate the temporal restriction of harmful processes, the mammalian circadian timing system gates hepatic 
production of bile acids to the absorptive phase, presumably to limit the cytotoxic action of these detergents to the time window when they are needed for lipid emulsification ( $\mathrm{Li}$ and Chiang 2014).

In mammals, nearly all cells harbor cell-autonomous and self-sustained circadian oscillators with a similar molecular makeup (see below). According to current belief these numerous clocks are synchronized by a central pacemaker in the suprachiasmatic nucleus (SCN), two small assemblies of neurons located above the optical chiasma in the ventral hypothalamus (for review, see Dibner et al. 2010; Partch et al. 2014). The SCN keeps phase coherence for infinite time periods, as manifested by the persistent rhythmic behavior of rodents kept in constant darkness (DD) (Sharma and Chandrashekaran 1998). However, in mice the period length generated by the SCN in DD is somewhat shorter than $24 \mathrm{~h}$, and the SCN must therefore be phase-adjusted by the photoperiod by a few minutes every day to remain in resonance with geophysical time. This is accomplished primarily through photic information transmitted from the retina to the SCN through the retinohypothalamic tract, resulting in the activation of cAMP response element-binding protein (CREB) in postsynaptic SCN neurons. CREB then stimulates the transcription of Period (Per) genes and thereby resets the phase of the SCN (for details see Meijer and Schwartz 2003; Ramkisoensing and Meijer 2015). In turn, the SCN is thought to synchronize the oscillators in peripheral tissues through a wide variety of indirect and more direct pathways, depending on blood-borne signals, feedingfasting rhythms, and, somewhat surprisingly, body temperature rhythms (Brown et al. 2002; Buhr et al. 2010; Saini et al. 2012). All in all, the SCN can orchestrate overt rhythmic outputs in behavior by systemic cues directly affecting the physiological process in question, by systemic signals that synchronize local clocks in peripheral tissues, or by a combination of the two.

\section{MOLECULAR MAKEUP AND SYNCHRONIZATION OF MAMMALIAN CIRCADIAN OSCILLATORS}

We cannot claim to understand how the molecular clockwork circuitry of mammals generates cycles in gene expression of $\sim 24 \mathrm{~h}$. Nonetheless, research conducted during the past two decades by many groups has revealed genes that are essential for proper circadian timing. Functional redundancies have rendered the genetic dissection of clock genes challenging. Indeed, to this date there is only a single gene, Bmall, whose disruption renders mice immediately arrhythmic when they are kept in DD (Bunger et al. 2000). For all of the other core clock genes there are at least two functionally redundant isoforms, and strong phenotypes are only observed in mice with multiple loss-of-function alleles (Buhr and Takahashi 2013). Detailed knowledge about the current state of understanding of mammalian circadian oscillators can be gathered from several excellent review articles (e.g., Baggs and Hogenesch 2010; Buhr and Takahashi 2013;
Partch et al. 2014). Figure 1 depicts skeleton models of circadian oscillators in SCN neurons and peripheral cell types with focus on the clock components that are relevant for the remaining part of this article. In both SCN neurons and peripheral cell types the molecular oscillators consist of two coupled feedback loops. In the canonical feedback loop, protein complexes with a molecular mass of $\sim 2 \mathrm{MDa}$ containing cryptochromes (CRY1 and CRY2), period proteins (PER1 and PER2), and about 30 additional polypeptides bind to and repress activator complexes harboring heterodimers of the basic helixloop-helix transcription factors CLOCK and BMAL1 (Kim et al. 2014, 2015; RP Aryal and CJ Weitz, unpubl. results). The latter stimulate transcription of $\mathrm{Per}$ and Cry genes, and when PER-CRY complexes reach a certain concentration they shut down their own expression by removing the CLOCK-BMAL1 complexes from the E-box sequences within promoters and enhancers of Per and Cry genes (Ye et al. 2014). A second feedback loop, orchestrated by nuclear orphan receptors of the REV-ERB (NR1D) and ROR (NR1F) families, drives the rhythmic transcription of Bmall and Clock. In this transcriptional circuitry REV-ERB $\alpha$ (NR1D1) and REV-ERB $\beta$ (NR1D2) repressors compete with ROR $\alpha$ (NR1F1), ROR $\beta$ (NR1F2, neuron-specific), and ROR $\gamma$ (NR1F3) for the binding to RORE elements in the Bmall and Clock promoters. As Rev-erb $\alpha$ and Rev-erb $\beta$ are direct targets of CLOCK-BMAL1 complexes, the canonical and secondary feedback loops are tightly coupled. Although the two linked feedback loops are operative in SCN neurons and peripheral tissues, there are several noteworthy differences between central and peripheral clocks.

1. CLOCK depletion abolishes circadian rhythm generation in peripheral organ slices kept in vitro or cultured cells (DeBruyne et al. 2007b), but not in cultured SCN slices, in which NPAS2, a closely related CLOCK homolog, can substitute for the latter (Debruyne et al. 2006, 2007a).

2. In the SCN of BMAL1-deficient neurons, Perl and Per 2 transcription is frozen at nadir values (Bunger et al. 2000). In contrast, in the livers of mice with BMAL1 - or CLOCK-depleted hepatocytes, PER2 expression continues to oscillate with amplitudes and magnitudes similar to those observed in hepatocytes of wild-type animals, and rhythmic PER1 accumulation shows only a moderate diminution in magnitude (Debruyne et al. 2006; Kornmann et al. 2007a,b). Therefore, circadian Per 2 transcription in the livers of intact animals must be driven mostly by systemic cues, sensed by transcription factors other than CLOCK-BMAL1 complexes. Some of these transcription factors have yet to be identified, but we propose that they functionally compete with CLOCKBMAL1 complexes (see Conclusion and Speculation: Driven versus Self-Sustained Oscillators).

3. The expression of the neuron-specific ROR $\beta$ isoforms in the SCN constitutes a further difference between the central pacemaker and nonneuronal peripheral clocks (Schaeren-Wiemers et al. 1997). 

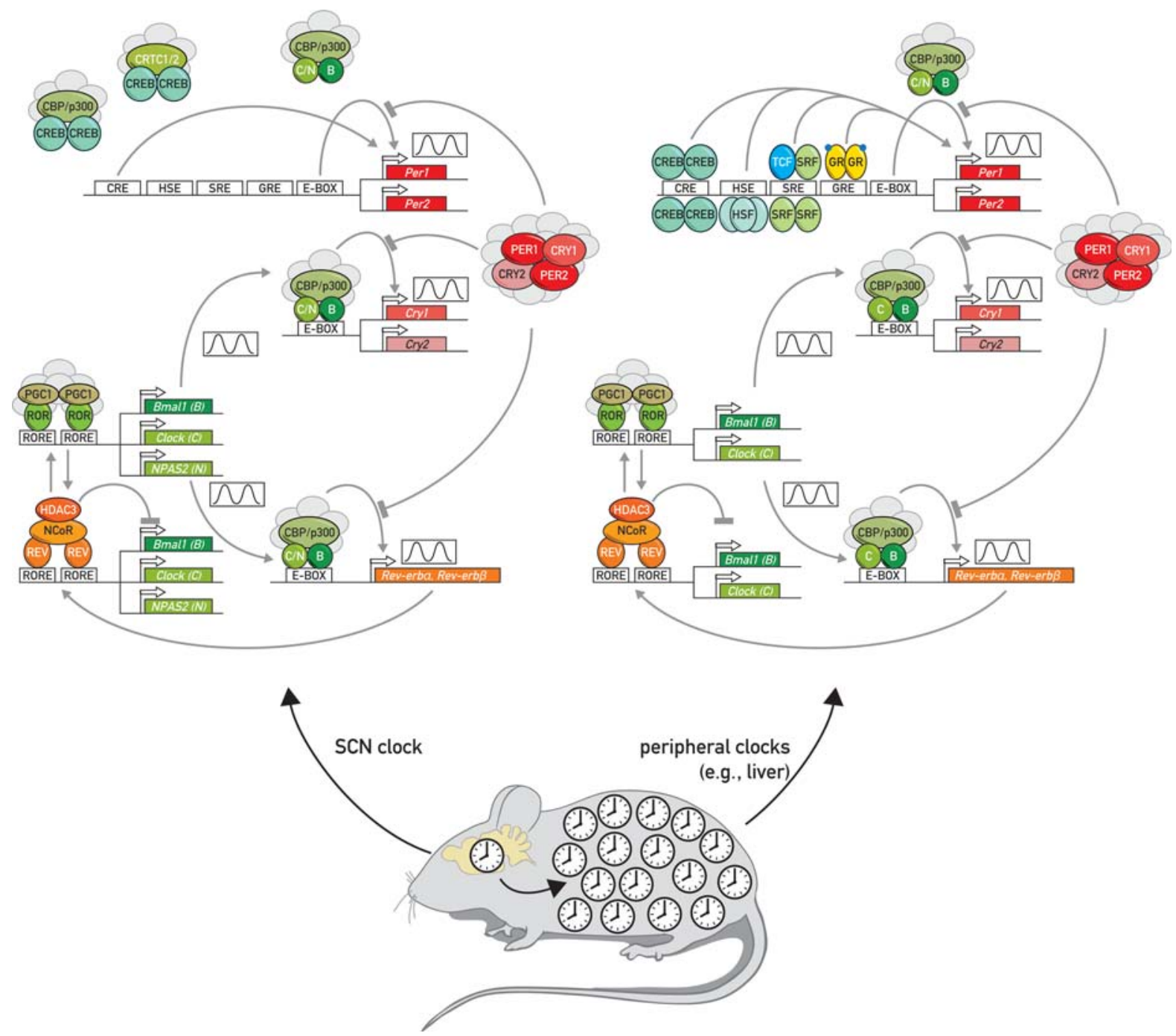

Figure 1. Circadian oscillators in suprachiasmatic nucleus $(\mathrm{SCN})$ neurons and peripheral cell types. Circadian oscillators have a similar makeup in SCN neurons and peripheral cell types. However, there are some interesting differences in how they function in the SCN and peripheral organs, such as the liver (see text). In the SCN, the core clock transcription factors CLOCK (or its paralog NPAS2, which can functionally replace CLOCK in the SCN) and BMAL1 are absolutely required for the circadian expression of the core clock repressors PER1 and PER2. Moreover, cAMP response element-binding protein (CREB)-related immediate early transcription factors (IEFs) and their coactivator complexes are probably the major transcriptional regulators synchronizing the SCN oscillators. In contrast, clocks in peripheral tissues are mainly driven by IEFs such as CREB (and paralogs), heat shock transcription factor 1 (HSF1), serum response factor (SRF), and glucocorticold receptor (GR), which sense various systemic signals (represented by an arrow pointing from the brain to the periphery). For example, in mice hepatocyte clocks continue to tick in the absence of CLOCK and BMAL1, as long as they receive systemic signals from the SCN or environmental cycles (see Fig. 4). Only when tissue slices are kept in tissue culture (i.e., in the absence of cyclic systemic cues), do the endogenous clocks depend on CLOCK-BMAL1 heterodimers (DeBruyne et al. 2007b; Kornmann et al. 2007a).

4. Cellular circadian oscillators are tightly coupled within the SCN, whereas they may or may not be coupled in the various peripheral tissues (Mohawk and Takahashi 2011; O’Neill et al. 2013).

5. The synchronization pathways are obviously different in SCN and peripheral cells. Light-dark cycles are clearly the major Zeitgebers for the central pacemaker, whereas feeding-fasting rhythms are the dominant phase resetting cues for most peripheral organs. In addition, glucocorticoid signaling, daily polymerization cycles of the actin cytoskeleton, and body temperature rhythms - all directly or indirectly depending on the $\mathrm{SCN}$ - participate in the synchronization of peripheral clocks. Remarkably, the SCN is largely insensitive to the signals it uses to phase-entrain peripheral clocks. Thus, it is weakly influenced, at best, by feeding-fasting rhythms (Damiola et al. 2000), temperature cycles (Buhr et al. 2010), or glucocorticoid signaling (Balsalobre et al. 2000). With regard to the latter, SCN neurons are among the few cell types in adult mice and rats that do not express the glucocorticoid receptor (GR) (Rosenfeld et al. 1988; Balsalobre et al. 2000). 


\section{THE SEARCH FOR SYSTEMIC TIMING CUES BY CANDIDATE APPROACHES: FOOD AND GLUCOCORTICOIDS}

As mentioned in the introductory section, the $\mathrm{SCN}$ can drive overt rhythms in behavior and physiology by controlling systemic cues, such as neuronal and humoral signals, body temperature rhythms, and metabolites, or by synchronizing local subsidiary oscillators in peripheral organs. Some of the systemic signals (i.e., glucocorticoid hormones) are involved in both pathways; that is, they orchestrate circadian outputs directly and participate in the synchronization of peripheral timekeepers. The pursuit of identifying rhythmic systemic signaling pathways is therefore of considerable heuristic and medical interest. We used both candidate approaches and unbiased screening strategies in the hope of finding diurnally active signaling mechanisms, and some of these will be reviewed below.

Daily rest-activity cycles drive feeding-fasting rhythms, and the latter engender daily oscillations in metabolism. Not surprisingly then, a large number of rhythmically expressed genes in the liver and other tissues associated with the intestinal tract fulfill different roles in the catabolism and/or anabolism of nutrients during the absorptive phase and the postabsorptive phase. If the circadian clocks in these organs were to anticipate these phases, it would make sense to synchronize them to metabolic cycles. It was thus obvious to examine whether feeding-fasting rhythms act as synchronization cues for peripheral clocks. Not only was this hypothesis verified, but it turned out that offering food exclusively during the light phase completely inverted the phase of circadian oscillators in most peripheral tissues (Damiola et al. 2000). As feeding-fasting rhythms had little impact on the phase of the SCN, they completely uncoupled peripheral from central clocks. This phase disconnection turned out to be extremely helpful in the analysis of signaling pathways potentially involved in the synchronization of peripheral oscillators. In fact, a major problem in such endeavors is the large redundancy of participating signaling mechanisms. In other words, the disruption of a single signaling pathway has minor effects at most on the steadystate phase, and this renders the assessment of its significance difficult. However, the phase-shifting kinetics is quite sensitive to the inactivation of a single signaling mechanism, and measuring the time required for peripheral oscillators to adapt to the newly imposed feedingfasting regimen can provide valuable information on whether or not an examined pathway is relevant in vivo. If the disruption of a given pathway accelerates phaseshifting kinetics, this pathway is likely to be controlled by the SCN. If, in contrast, the inactivation of a signaling route slows down phase-shifting kinetics, the investigated synchronization mechanism is probably participating in the phase entrainment by feeding-fasting cycles.

How can feeding and/or fasting synchronize peripheral clocks? Our knowledge on the involved molecular mechanisms is scrappy at best and entirely based on candidate approaches. Among the culprits one would expect metabolites and their sensors, feeding-dependent hormones and their receptors, and, perhaps, neuronal signals transmitted from nutrient-sensing brain areas to peripheral organs via the peripheral nervous system. Several nutritional status-dependent signal sensors belonging to these classes have been proposed, including AMP-dependent kinase (AMPK) (Lamia et al. 2009), insulinphosphatidylinositol 3-kinase (PI3 K) (Chaves et al. 2014), insulin (Yamajuku et al. 2012), and the two $\mathrm{NAD}^{+}$-sensing enzymes Sirtuin 1 (SIRT1) (Asher et al. 2008; Nakahata et al. 2008) and PARP-1 (Asher et al. 2010). The phase-shifting kinetics outlined above have been compared for Parp- $1^{-/-}$and Parp- $1^{+/+}$mice, and these experiments have confirmed the role of PARP-1 in the phase entrainment of liver oscillators by feedingfasting cycles (Asher et al. 2010).

The plasma levels of glucocorticoid hormones - cortisol in humans and corticosterone in laboratory rodentshave long been known to accumulate with an about 10fold daily amplitude. Moreover, dexamethasone, a potent GR agonist, efficiently synchronizes circadian oscillators in cultured fibroblasts, probably mainly through the immediate early activation of Perl (Balsalobre et al. 2000). We thus wondered whether glucocorticoid signaling also participates in the synchronization of peripheral clocks in vivo. Experiments with mice harboring a hepatocytespecific disruption of the $G r$ gene first looked disappointing, as their circadian liver gene expression was nearly identical to that of wild-type mice (Balsalobre et al. 2000). However, as aforementioned the steady-state phase is not very sensitive to a single phase entrainment pathway, as many others are still operative. Indeed, when recording phase-shifting kinetics after inversion of the feeding-fasting regimen we noticed that the $G r$-deficient hepatocytes adapted their phase more rapidly to daytime feeding than their wild-type counterparts (Le Minh et al. 2001). Hence, signaling through the GR is likely to participate in the synchronization of hepatocyte oscillators, and it depends on cues directly controlled by the SCN rather than on feeding-fasting rhythms (Le Minh et al. 2001).

The recording of phase-shifting kinetics described above for the pathways involving GR and PARP-1 has been both labor-intensive and unpleasant. In fact, it involved the analysis of rhythmically expressed mRNAs from multiple mice of two genetic backgrounds killed at 4-h intervals around the clock during multiple days. Moreover, because of interindividual variabilities, the temporal resolution is limited in such experiments, and the contribution of pathways whose elimination results only in small differences would hence be difficult to evaluate. Recently we have succeeded in establishing a technology allowing us to monitor bioluminescence cycles produced by peripheral organs expressing circadian luciferase reporter genes in real time and in freely moving animals (Saini et al. 2013). This method, dubbed RT-Biolumicording (Fig. 2), renders the recording of phase-shifting experiments with a high temporal resolution very easy. Importantly, it permits the temporal determination of gene expression in SCN-lesioned, 
A

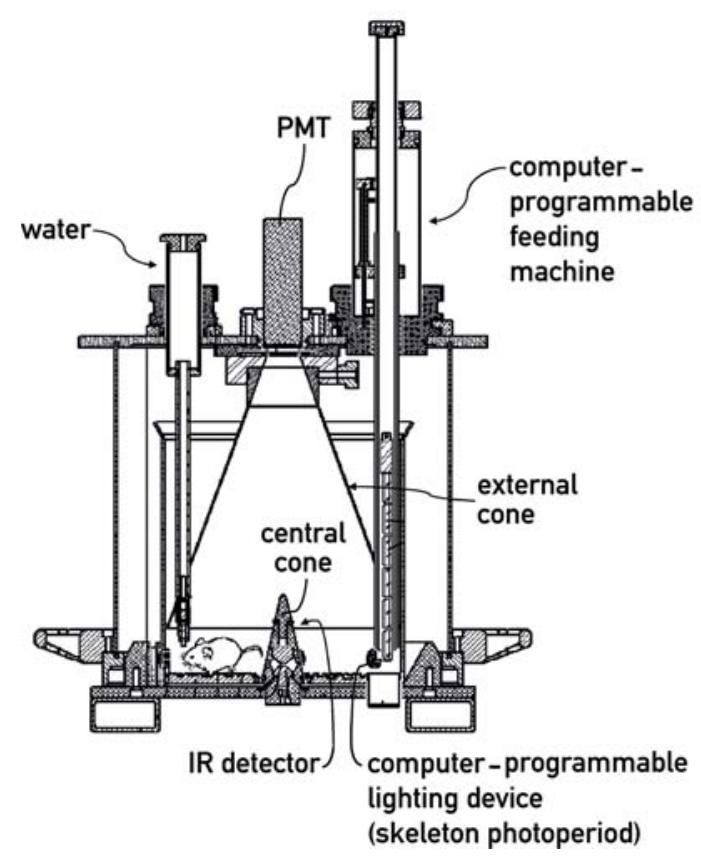

B

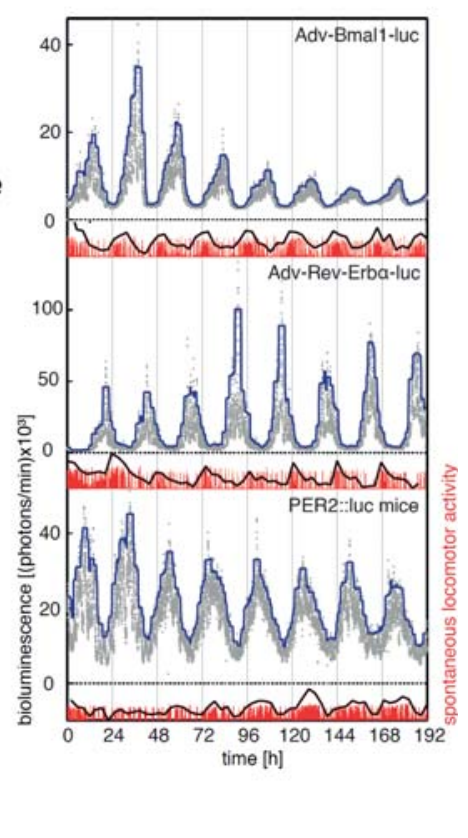

Figure 2. Real-time monitoring of circadian gene expression in freely moving mice. (A) The RT-Biolumicorder consists of a cylindrical, photon-proof cage equipped with light-reflecting walls, a photomultiplier tube (PMT) with a programmable shutter, a light-emitting diode (LED) light source, a programmable feeding machine, a water reservoir, and a passive infrared recording device (PIR) under the central cone. Photons emitted by a mouse expressing a luciferase reporter gene are guided to the PMT and counted. The PIR monitors spontaneous locomotor activity. If necessary, the SCN can be synchronized by skeleton photoperiods (i.e., two LED light pulses given at $12-\mathrm{h}$ intervals). During the light pulses the PMTs are automatically protected by a programmable shutter. $(B)$ Bioluminescence recordings of three mice expressing different luciferase reporter genes. Adenoviral vectors containing Bmall-luciferase (Ad-Bmal1-luc) and Rev-Erb $\alpha$-luciferase (Ad-Rev-Erb $\alpha$-luc) reporters were transduced into the liver of hairless mice by tail vein injection. Per2::luc mice (strain background C57B6) express a PER2-luciferase fusion protein in all cells. However, they were depilated only in a small area on top of the liver, and a large fraction of the bioluminescence was thus contributed by this organ. The mice had Alzet pumps delivering constant amounts of luciferin implanted into their intraperitoneal cavity. The spontaneous locomotor activity is depicted in red. ( $A, B$, Reprinted, with permission, from Saini et al. 2013, Cold Spring Harbor Laboratory Press.)

behaviorally arrhythmic mice. Obviously, the establishment of time series in RNA or protein accumulation by conventional techniques (i.e., by comparing extracts of mice killed at different time intervals) is impossible in such animals, because they are behaviorally arrhythmic. By employing RT-Biolumicording we have, for the first time, shown that the SCN is indeed required to establish phase coherence between peripheral oscillators, but that within the liver the hepatocyte clocks remain synchronized during months after the SCN had been ablated ( $\mathrm{P}$ Gos, T Curie, Y Emmenegger, P Franken, and U Schibler, unpubl. results).

\section{THE SEARCH FOR SYSTEMIC TIMING CUES BY UNBIASED APPROACHES: BODY TEMPERATURE AND ACTIN DYNAMICS}

As outlined above, circadian gene expression in peripheral cell types can be orchestrated either by local oscillators or by systemic cues depending on the SCN and/or on daily changes in the environment. How can one discriminate between genes whose cyclic expression is driven by local clocks or systemic signals? To address this problem we developed a transgenic mouse model with con- ditionally active liver clocks exclusively in hepatocytes. In the presence of Doxycycline (Dox), the local liver clocks are ticking, whereas in the absence of Dox they are arrested (Kornmann et al. 2007a,b). Genome-wide transcriptome profiling at 4-h intervals around the clock for $2 \mathrm{~d}$ were then performed on liver RNA prepared from mice fed with normal chow or Dox-containing chow. Using stringent criteria we identified about 350 diurnally expressed mRNAs, of which about 60 had similar accumulation profiles in the presence and absence of Dox. Given that local, self-sustained liver clocks were not operative in the absence of Dox, the diurnal expression of the Dox-independent genes was supposedly driven by systemic signals rather than hepatocyte clocks. The identity of Dox-independent genes suggested several pathways that may participate in the systemic control of their rhythmic expression. Surprisingly, Per2, a bona fide core clock gene, was among the genes whose expression patterns did not depend on functional hepatocyte oscillators. This observation immediately alludes to a mechanism that could account for the synchronization of hepatocyte clocks. According to this mechanism, Per2 serves as both a major sensor for cyclic signaling cues and a core clock component. The latter has been verified in experiments 
with liver explants from PER2::luciferase mice (Yoo et al. 2004) with Dox-dependent BMAL1-expression. When these were placed into culture media with or without Dox, PER2::luciferase bioluminescence cycles could only be observed in the presence of Dox. Therefore, the rhythmic expression of the Per2::luciferase fusion gene requires a functional local clock in the absence of the rhythmic systemic cues operative in vivo.

Among the systemically regulated genes, we also found genes whose expression had previously been shown to be temperature-dependent. These included several genes encoding heat shock proteins (also known as chaperones that fold nascent or denatured proteins) and cold-inducible RNA-binding proteins (e.g., CIRP). In a completely independent unbiased approach, dubbed differential display of DNA-binding proteins (DDDP), we identified heat shock factor 1 (HSF1) as a transcription factor shuttling from the cytoplasm to the nucleus in a diurnal fashion, with a phase consistent with body temperature rhythms (Reinke et al. 2008). Mouse body temperature smoothly oscillates between $\sim 35^{\circ} \mathrm{C}$ and $38^{\circ} \mathrm{C}$, and one might wonder whether such small differences can indeed synchronize circadian oscillators. Previously, Brown and collaborators have already showed that square-wave temperature steps $\left(12 \mathrm{~h} 33^{\circ} \mathrm{C} / 12 \mathrm{~h} 37^{\circ} \mathrm{C}\right)$ could efficiently phase-entrain circadian gene expression in cultured RAT1 fibroblasts, and that smooth simulated body temperature oscillations could sustain phase coherence of presynchronized cells (Brown et al. 2012). Subsequent studies with more sophisticated recording technologies have conclusively shown that smooth simulated mouse body temperature rhythms oscillating between $35^{\circ} \mathrm{C}$ and $38^{\circ} \mathrm{C}$ can efficiently synchronize circadian gene expression de novo. As already noticed for glucocorticoids and feeding-fasting rhythms, the phase of the SCN appears to be resilient to temperature rhythms, both in animals (Brown et al. 2002) and in organotypic cultures (Buhr et al. 2010). The phase of HSF1-deficient fibroblasts adapts more sluggishly to phase shifts in temperature rhythms than that of HSF-proficient cells. Hence, HSF must participate in the synchronization of peripheral oscillators by body temperature oscillations. However, because even HSF1-depleted cells eventually synchronize to altered temperature cycles, additional temperature-sensitive regulators must be involved in this process (Fig. 3; Saini et al. 2012).

The gene specifying the cold-inducible RNA-binding protein CIRP reaches maximal levels in the liver during the resting phase (i.e., when body temperature is minimal). Rhythmic CIRP accumulation can be mimicked in cultured cells exposed to simulated body temperature cycles (Morf et al. 2012). In CIRP-depleted fibroblasts, the amplitude of circadian gene expression is dampened, perhaps because Clock mRNA is exported less efficiently from the nucleus to the cytoplasm in these cells (Morf et al. 2012). As expected from this reduction in amplitude, CIRP-depleted fibroblasts adapt more rapidly to phase shifts in temperature cycles than CIRP-proficient cells. In vivo, however, hepatocyte clocks adapt more slowly to altered light-dark cycles in the former than in the latter.

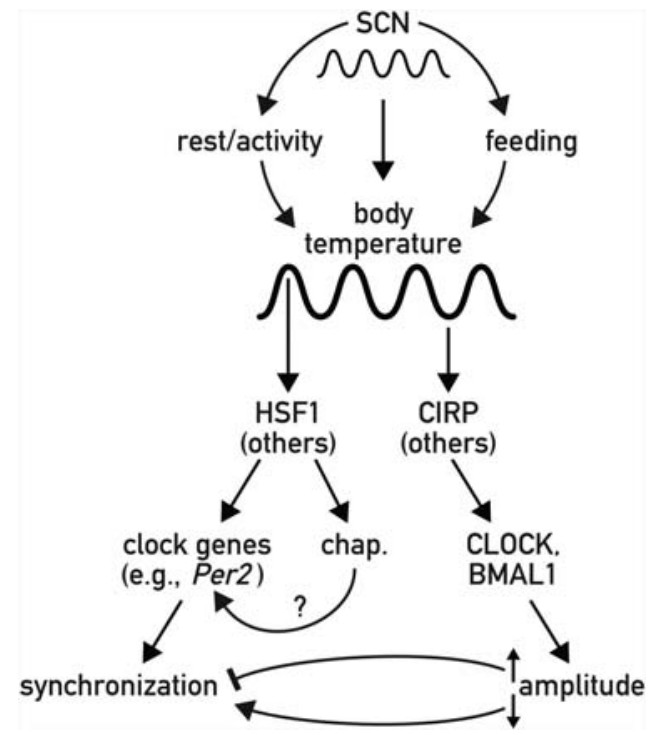

Figure 3. Modulation of circadian gene expression by body temperature rhythms. Daily body temperature oscillations are influenced directly and indirectly by the SCN. In mice kept at $24^{\circ} \mathrm{C}$ they fluctuate between $\sim 35^{\circ} \mathrm{C}$ and $38^{\circ} \mathrm{C}$ - that is, with an amplitude of $\sim 1.5^{\circ} \mathrm{C}\left(\left[38^{\circ} \mathrm{C}-35^{\circ} \mathrm{C}\right] / 2\right)$. Such temperature rhythms, when imposed on cultured fibroblasts, are sufficient to drive robust oscillations in the expression of heat- and coldinducible genes and to synchronize circadian gene expression. Although HSF1 is involved in the synchronization of circadian clocks, the cold-inducible RNA-binding protein CIRP participates in the modulation of CLOCK and BMAL1 accumulation and thereby influences the amplitude of circadian gene expression. CIRP loss of function attenuates the expression of CLOCK-BMAL1 target genes (such as Rev-erb $\alpha$ and $D b p$ ) in cultured fibroblasts, but enhances the expression of these genes in the liver of mice (F Sinturel and U Schibler, unpubl. results). Hence, the effect of CIRP on the amplitude and magnitude of circadian gene expression may be cell type-specific. chap., chaperones (i.e., heat shock proteins).

Conceivably, CIRP modulates the amplitude and phaseshifting capacity in a cell type-specific manner (F Sinturel, P Gos, and U Schibler, unpubl. results). Whereas the daily expression cycles of heat shock mRNAs are driven primarily by HSF1-dependent transcriptional mechanisms, the diurnal rhythm of Cirp mRNA accumulation is regulated at the posttranscriptional level, most probably by a temperature-dependent efficiency of Cirp pre-mRNA splicing (I Gotic and U Schibler, unpubl. results).

Elegant parabiosis experiments with intact and SCNlesioned mice performed in Eric Bittman's laboratory indicated that blood-borne signals can synchronize clocks at least in some peripheral organs (Guo et al. 2005). To identify signaling pathways relying on diurnally active blood-borne factors we developed a novel screening procedure, dubbed STAR-PROM (for synthetic tandem repeat promoter screening). This technology can identify immediate early transcription factors (IEFs), which usually play the endgame in a signal transduction cascade, even if their DNA-binding specificity is unknown. In our initial STAR-PROM screening we used plasma samples harvested at 3-h intervals from healthy human subjects to uncover diurnally activated IEFs in U2OS cells (Gerber 
et al. 2013). The RhoA-MRTF-SRF pathway was one of the diurnally active signaling pathways revealed by STAR-PROM screening. In this pathway, initially discovered by Richard Treisman and coworkers (for review, see Posern and Treisman 2006), the activation of the RhoA GTPase promotes the polymerization of globular actin (g-actin) into filamentous actin (f-actin). As g-actin acts as a stoichiometric repressor of myocardin-related transcription factors (MRTFs), actin polymerization releases MRTF into the active nuclear form, which is recruited as a coactivator to prebound SRF and triggers transcription of SRF target genes. Per2 is among these genes (Gerber et al. 2013; Esnault et al. 2014), and this offers a plausible mechanism for the synchronization of peripheral clocks by the MRTF-SRF pathway. Because the STAR-PROM experiments were conducted with cultured cells, it was important to scrutinize whether the MRTF pathway is relevant in vivo. To this end we examined the nuclear accumulation of MRTF and the fractions of $g$ - and f-actin around the clock and found that these parameters followed a robust circadian rhythm. Moreover, the activation of MRTFs in cultured cells by the drugs cytochalasin and jasplakinolide stimulates Per 2 expression and thereby synchronizes circadian clocks (Esnault et al. 2014; P Gos, G Rando, and U Schibler, unpubl. data). Perhaps more importantly, in vivo SRF-deficient hepatocyte clocks synchronize more rapidly to a feeding regimen whose phase is in conflict with that of the SCN than wild-type hepatocytes. Therefore, we consider likely that signaling through the MRTF-SRF pathway participates in the phase entrainment of peripheral oscillators in vivo and that it does so in a feeding-independent, SCNdependent fashion (P Gos, A Nordheim, and U Schibler, unpubl. results).

\section{CONCLUSION AND SPECULATION: DRIVEN VERSUS SELF-SUSTAINED OSCILLATORS}

Many different pathways depending on various signals controlled by the SCN and environmental factors participate in the synchronization of clocks in peripheral cell types. In vivo studies have concentrated primarily on the liver, but it is well possible, if not likely, that different tissues can be phase-entrained by distinct pathways. A striking observation made in several laboratories is that the rhythmic hepatic expression of the four negative limb members such as PER1, PER2, CRY1, and CRY2 only marginally depends on the positive members such as CLOCK and BMAL1. Furthermore, in the livers of Clock knockout mice the temporal accumulation patterns of $C r y$ and Per mRNAs do not follow those expected from the simple feedback loop depicted in Figure 1. Thus, Cryl and Cry 2 transcripts accumulate at constitutively high, rather than low, levels in these animals. Perl mRNA oscillates with high amplitude and a magnitude that is about threefold higher than that observed in wild-type mice. Per 2 mRNA accumulation is only affected during the nadir times of expression in these animals (Debruyne et al. 2006). In contrast, the expression of the direct CLOCK-BMAL1 target genes $\mathrm{Dbp}$ (a clock output regulator gene) and Rev-erb $\alpha$ (a regulatory gene of the secondary feedback loop) is dramatically down-regulated in both Clock and Bmall knockout mice (Bunger et al. 2000; Debruyne et al. 2006). Within the positive limb, Bmal1 transcripts are expressed at higher than wild-type levels in Clock knockout mice, presumably because of the strongly diminished REV-ERB $\alpha$ accumulation in these animals. However, despite increased mRNA amounts, hepatic BMAL1 protein levels are strongly reduced in these mice (Debruyne et al. 2006). Supposedly BMAL1 is stabilized by its association with CLOCK, and the depletion of its dimerization partner promotes its degradation. How can we reconcile the apparently conflicting observation that the depletion of CLOCK and BMAL1 has only minor effects on circadian PER and CRY accumulation, yet dramatically affects the cyclic expression of clock-controlled genes? We propose that in liver the circadian transcription of Per1 and Per 2 is regulated by alternate episodes of transactivation by diurnally activated transcription factors (including the IEFs such as SRF, GR, and HSF1, as well as SP1 [M. Demarque and U Schibler, unpubl. data]) and CLOCK-BMAL1 heterodimers. Because the magnitude of Per 2 mRNA accumulation is not reduced by the depletion of CLOCK and BMAL1, the activation mechanisms involving IEFs (and SP1) and CLOCK-BMAL1 are likely to be mutually exclusive. Otherwise put, the IEFs (and SP1) stimulate Per 2 transcription more efficiently in the absence of CLOCK and BMAL1 and thereby compensate for the loss of activation by CLOCK-BMAL1 heterodimers in mutant animals whose hepatocytes are deficient for these core clock transcription factors. Hepatic Perl transcripts still accumulate rhythmically, albeit with a delayed phase, in the absence of CLOCK (Debruyne et al. 2006) and BMAL1 (Kornmann et al. 2007a). Surprisingly, however, the magnitude of Perl mRNA accumulation is decreased in BMAL1-depleted and increased in CLOCK-deficient hepatocytes. Cryl mRNA accumulation is constitutively high in Clock knockout mice, which, owing to the redundant functions of CLOCK and BMAL1 in the SCN, are still behaviorally rhythmic. Hence in wild-type mice CLOCK and BMAL1 must directly or indirectly suppress Cryl mRNA accumulation during the nadir times. In spite of these alterations in mRNA expression in Clock knockout mice and mice with a liver-specific Bmal1 knockdown, all four negative limb members PER1, PER2, CRY1, and CRY2 accumulate with a similar phase and amplitude (and an only moderate reduction in magnitude) in these animals (Debruyne et al. 2006; Kornmann et al. 2007a). Beautiful biochemical work performed in the laboratory of Charles Weitz may offer a plausible explanation for this enigma. The biochemical analysis of PER2-containing protein complexes from liver nuclear extracts showed that CRY and PER proteins form stable complexes with a molecular mass of $\sim 2 \mathrm{MDa}$, and that smaller complexes or monomers are undetectable on blue native gels (Kim et al. 2014, 2015; RP Aryal and CJ Weitz, unpubl. results). We thus 
A
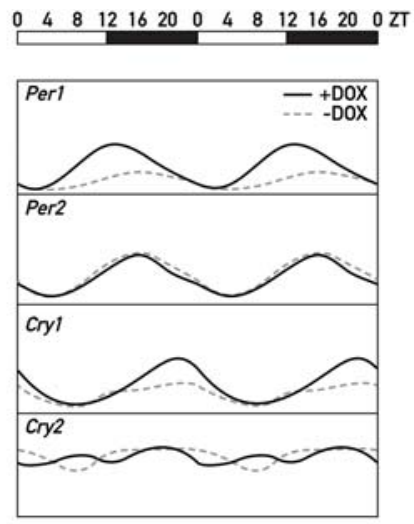

B

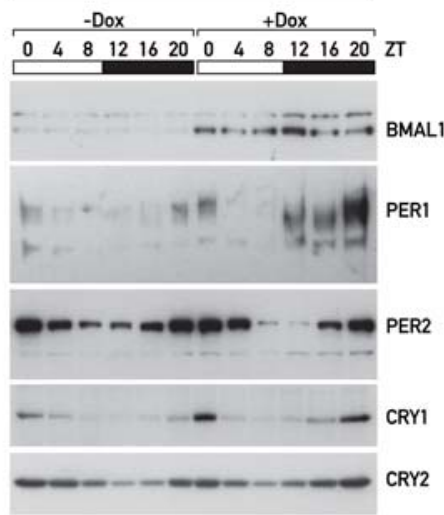

D
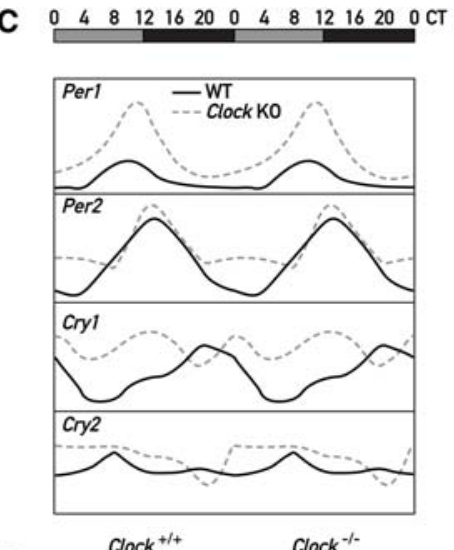

$2 \quad 610141822 \quad 2 \quad 6 \quad 610141822$ CT

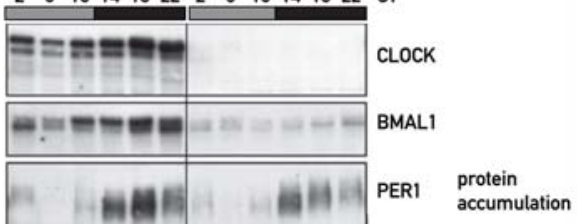

E

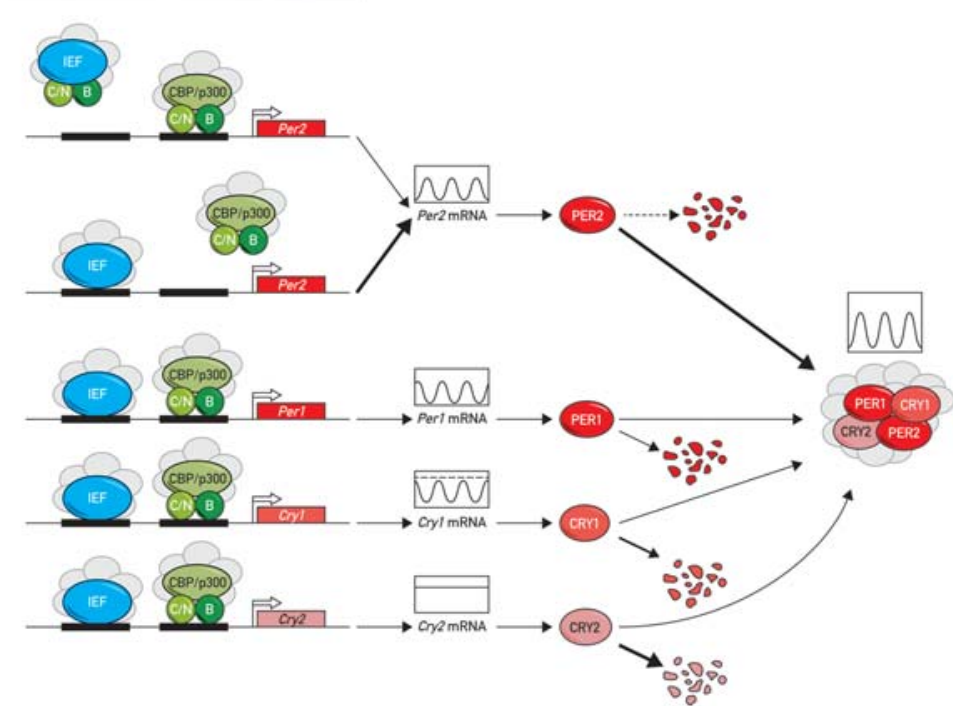

Figure 4. Systemic and core clock mechanisms are interdigitated in the liver. Circadian accumulation of PER and CRY mRNAs $(A, C)$ and proteins $(B, D)$ in mice with a hepatocyte-specific knockdown of BMAL1 expression $(A, B)$ and a body-wide knockout of CLOCK $(C, D)$. Note that all mice analyzed in $A-D$ are behaviorally rhythmic. The data shown in $A$ and $B$ have been obtained with mice kept under a 12-h light/12-h dark regimen (illustrated by the white and black bars on top of the panels). The data depicted in $C$ and $D$ have been obtained with mice kept during $1 \mathrm{~d}$ in constant darkness (subjective days and nights are represented in gray and black bars, respectively, above the panels). (A) Double plots of hepatic Per and Cry mRNA accumulation curves in mice with normal (+DOX) and attenuated BMAL1 ( - DOX) expression in the liver. $(B)$ Immunoblot data showing the temporal accumulation of BMAL1, PER, and CRY proteins in mice with normal $(+\mathrm{DOX})$ and attenuated $(-\mathrm{DOX})$ BMAL1 expression in the liver. $(C)$ Smoothened double plots of hepatic Per and Cry mRNA accumulation curves in the livers of wild-type (WT) mice and Clock knockout mice. (D) Immunoblot data showing the temporal accumulation of CLOCK, BMAL1, and PER and CRY proteins in the livers of WT mice and Clock knockout mice. (E) Schematic representation of circadian PER and CRY expression in liver (and, perhaps, other peripheral organs). As CLOCK and BMAL1 are not required for the rhythmic accumulation of PER and CRY proteins in the liver (see $B, D$ ), the rhythmic expression of these proteins must be driven by immediate early transcription factors (IEFs) sensing diurnal systemic signals. Except for Per 2 expression, temporal mRNA accumulation cannot account for temporal protein accumulation. In fact, both Cry 1 and Cry 2 mRNAs are expressed at similar levels throughout the day in Clock knockout mice, yet the proteins encoded by these mRNAs show robustly circadian accumulation. We propose that cyclic Per2 transcription is regulated by the interdigitated action between CLOCK-BMAL heterodimers and rhythmically activated IEFs, and that PER2 is the rate-limiting component for the assembly of the large PER-CRY complexes. According to this model, PER1, CRY1, and CRY2 are synthesized in excess, and proteins not incorporated into the PER-CRY complexes are rapidly degraded. ( $A, B$, Adapted from Kornmann et al. 2007a; $C, D$, adapted from DeBruyne et al. 2006, with permission from Elsevier.) 
speculate that PER2 is the rate-limiting component for the formation of the 2-MDa complexes in the liver (and perhaps other peripheral organs). In contrast, CRY proteins and PER1 may be produced in excess, and surplus molecules not incorporated into large complexes may be rapidly degraded (Fig. 4). In summary, the major role of CLOCK-BMAL1 heterodimers in peripheral organs may be to regulate the rhythmic expression of clock-output regulators (e.g., the PAR bZip transcription factors DBP, TEF, and HLF [Gachon 2007]) or orphan nuclear receptors of the ROR or REV-ERB family (Preitner et al. 2002; Fang and Lazar 2015), rather than that of CRYs and PERs. This is quite different from their tasks in the SCN master pacemaker, where they strictly control Per and Cry gene transcription and thereby generate self-sustained rhythms of clock gene expression that lie at the basis of circadian behavior and physiology.

\section{ACKNOWLEDGMENTS}

We thank Charles J. Weitz, Harvard Medical School, for the communication of unpublished results, and Nicolas Roggli for the artwork. Work in the laboratory of U.S. was supported by the University of Geneva, the State of Geneva, the European Research Council (ERCAdG TimeSignal), the Swiss National Science Foundation (SNF 31-113565, SNF 31-128656/1 and the National Centres of Competence in Research [NCCR] program grant Frontiers in Genetics), the Louis Jeantet Foundation of Medicine, a Federation of European Biochemical Societies (FEBS) long-term postdoctoral fellowship to I.G., and a European Molecular Biology Organization (EMBO) long-term fellowship to F.S. Work in the laboratory of Paul Franken was supported by the University of Lausanne, the State of Vaud, the Swiss National Science Foundation (SNF 31003A-130825, and SNF 31003A-146694), the European Mouse Disease Clinic (EUMODIC) (Contract no.: 037188 supporting Y.E.), and postdoctoral fellowships of the Marie Curie IntraEuropean program (IEF-FP7-Project Number: 221254) and the Novartis Foundation to T.C.

\section{REFERENCES}

Asher G, Gatfield D, Stratmann M, Reinke H, Dibner C, Kreppel F, Mostoslavsky R, Alt FW, Schibler U. 2008. SIRT1 regulates circadian clock gene expression through PER2 deacetylation. Cell 134: 317-328.

Asher G, Reinke H, Altmeyer M, Gutierrez-Arcelus M, Hottiger MO, Schibler U. 2010. Poly(ADP-ribose) polymerase 1 participates in the phase entrainment of circadian clocks to feeding. Cell 142: 943-953.

Baggs JE, Hogenesch JB. 2010. Genomics and systems approaches in the mammalian circadian clock. Curr Opin Genet Dev 20: $581-587$.

Balsalobre A, Brown SA, Marcacci L, Tronche F, Kellendonk C, Reichardt HM, Schütz G, Schibler U. 2000. Resetting of circadian time in peripheral tissues by glucocorticoid signaling. Science 289: 2344-2347.

Brown SA, Zumbrunn G, Fleury-Olela F, Preitner N, Schibler U. 2002. Rhythms of mammalian body temperature can sustain peripheral circadian clocks. Curr Biol 12: 1574-1583.
Buhr ED, Takahashi JS. 2013. Molecular components of the mammalian circadian clock. Handb Exp Pharmacol 2013: $3-27$.

Buhr ED, Yoo SH, Takahashi JS. 2010. Temperature as a universal resetting cue for mammalian circadian oscillators. Science 330: 379-385.

Bunger MK, Wilsbacher LD, Moran SM, Clendenin C, Radcliffe LA, Hogenesch JB, Simon MC, Takahashi JS, Bradfield CA. 2000. Mop3 is an essential component of the master circadian pacemaker in mammals. Cell 103: 1009-1017.

Chaves I, van der Horst GTJ, Schellevis R, Nijman RM, Koerkamp MG, Holstege FCP, Smidt MP, Hoekman MFM. 2014. Insulin-FOXO3 signaling modulates circadian rhythms via regulation of clock transcription. Curr Biol 24: 1248-1255.

Damiola F, Le Minh N, Preitner N, Kornmann B, Fleury-Olela F, Schibler U. 2000. Restricted feeding uncouples circadian oscillators in peripheral tissues from the central pacemaker in the suprachiasmatic nucleus. Genes Dev 14: 2950-2961.

DeBruyne JP, Noton E, Lambert CM, Maywood ES, Weaver DR, Reppert SM. 2006. A clock shock: Mouse CLOCK is not required for circadian oscillator function. Neuron 50: 465-477.

DeBruyne JP, Weaver DR, Reppert SM. 2007a. CLOCK and NPAS2 have overlapping roles in the suprachiasmatic circadian clock. Nat Neurosci 10: 543-545.

DeBruyne JP, Weaver DR, Reppert SM. 2007b. Peripheral circadian oscillators require CLOCK. Curr Biol 17: R538R539.

DeBruyne JP, Weaver DR, Dallmann R. 2014. The hepatic circadian clock modulates xenobiotic metabolism in mice. J Biol Rhythms 29: 277-287.

Dibner C, Schibler U, Albrecht U. 2010. The mammalian circadian timing system: Organization and coordination of central and peripheral clocks. Annu Rev Physiol 72: 517-549.

Dodd AN, Salathia N, Hall A, Kévei E, Tóth R, Nagy F, Hibberd JM, Millar AJ, Webb AA. 2005. Plant circadian clocks increase photosynthesis, growth, survival, and competitive advantage. Science 309: 630-633.

Esnault C, Stewart A, Gualdrini F, East P, Horswell S, Matthews N, Treisman R. 2014. Rho-actin signaling to the MRTF coactivators dominates the immediate transcriptional response to serum in fibroblasts. Genes Dev 28: $943-958$.

Fang B, Lazar MA. 2015. Dissecting the Rev-erb $\alpha$ cistrome and the mechanisms controlling circadian transcription in liver. Cold Spring Harbor Symp Quant Biol 80: in press. doi:10.1101/sqb.2015.80.027508.

Gachon F. 2007. Physiological function of PARbZip circadian clock-controlled transcription factors. Ann Med 39: 562-571.

Gachon F, Firsov D. 2011. The role of circadian timing system on drug metabolism and detoxification. Expert Opin Drug Metab Toxicol 7: 147-158.

Gehring W, Rosbash M. 2003. The coevolution of bluelight photoreception and circadian rhythms. J Mol Evol 57(suppl 1): S286-S289.

Gerber A, Esnault C, Aubert G, Treisman R, Pralong F, Schibler U. 2013. Blood-borne circadian signal stimulates daily oscillations in actin dynamics and SRF activity. Cell 152: 492503.

Guo H, Brewer JM, Champhekar A, Harris RB, Bittman EL. 2005. Differential control of peripheral circadian rhythms by suprachiasmatic-dependent neural signals. Proc Natl Acad Sci 102: 3111-3116.

Kim JY, Kwak PB, Weitz CJ. 2014. Specificity in circadian clock feedback from targeted reconstitution of the NuRD corepressor. Mol Cell 56: 738-748.

Kim JY, Kwak PB, Gebert M, Duong HA, Weitz CJ. 2015. Purification and analysis of PERIOD protein complexes of the mammalian circadian clock. Methods Enzymol 551: $197-210$

Kornmann B, Schaad O, Bujard H, Takahashi JS, Schibler U. 2007a. System-driven and oscillator-dependent circadian transcription in mice with a conditionally active liver clock. PLoS Biol 5: e34. 
Kornmann B, Schaad O, Reinke H, Saini C, Schibler U. 2007b. Regulation of circadian gene expression in liver by systemic signals and hepatocyte oscillators. Cold Spring Harb Symp Quant Biol 72: 319-330.

Lamia KA, Sachdeva UM, DiTacchio L, Williams EC, Alvarez JG, Egan DF, Vasquez DS, Juguilon H, Panda S, Shaw RJ, et al. 2009. AMPK regulates the circadian clock by cryptochrome phosphorylation and degradation. Science 326: 437-440.

Le Minh N, Damiola F, Tronche F, Schütz G, Schibler U. 2001. Glucocorticoid hormones inhibit food-induced phaseshifting of peripheral circadian oscillators. EMBO J 20: $7128-7136$.

Li T, Chiang JY. 2014. Bile acid signaling in metabolic disease and drug therapy. Pharmacol Rev 66: 948-983.

Meijer JH, Schwartz WJ. 2003. In search of the pathways for light-induced pacemaker resetting in the suprachiasmatic nucleus. J Biol Rhythms 18: 235-249.

Mohawk JA, Takahashi JS. 2011. Cell autonomy and synchrony of suprachiasmatic nucleus circadian oscillators. Trends Neurosci 34: 349-358.

Mohr W, Vagner T, Kuypers MM, Ackermann M, Laroche J. 2013. Resolution of conflicting signals at the single-cell level in the regulation of cyanobacterial photosynthesis and nitrogen fixation. PLoS One 8: e66060.

Morf J, Rey G, Schneider K, Stratmann M, Fujita J, Naef F, Schibler U. 2012. Cold-inducible RNA-binding protein modulates circadian gene expression posttranscriptionally. Science 338: $379-383$.

Nakahata Y, Kaluzova M, Grimaldi B, Sahar S, Hirayama J, Chen D, Guarente LP, Sassone-Corsi P. 2008. The NAD ${ }^{+}$dependent deacetylase SIRT1 modulates CLOCK-mediated chromatin remodeling and circadian control. Cell 134: 329340 .

O’Neill JS, Maywood ES, Hastings MH. 2013. Cellular mechanisms of circadian pacemaking: Beyond transcriptional loops. Handb Exp Pharmacol 2013: 67-103.

Partch CL, Green CB, Takahashi JS. 2014. Molecular architecture of the mammalian circadian clock. Trends Cell Biol 24: 90-99.

Posern G, Treisman R. 2006. Actin' together: Serum response factor, its cofactors and the link to signal transduction. Trends Cell Biol 16: 588-596.

Preitner N, Damiola F, Lopez-Molina L, Zakany J, Duboule D, Albrecht U, Schibler U. 2002. The orphan nuclear receptor REV-ERB $\alpha$ controls circadian transcription within the positive limb of the mammalian circadian oscillator. Cell 110: 251-260.

Ramkisoensing A, Meijer JH. 2015. Synchronization of biological clock neurons by light and peripheral feedback systems promotes circadian rhythms and health. Front Neurol 6: 128.
Reinke H, Saini C, Fleury-Olela F, Dibner C, Benjamin IJ, Schibler U. 2008. Differential display of DNA-binding proteins reveals heat-shock factor 1 as a circadian transcription factor. Genes Dev 22: 331-345.

Rosenfeld P, Van Eekelen JA, Levine S, De Kloet ER. 1988. Ontogeny of the type 2 glucocorticoid receptor in discrete rat brain regions: An immunocytochemical study. Brain Res 470: $119-127$.

Sadacca LA, Lamia KA, deLemos AS, Blum B, Weitz CJ. 2011. An intrinsic circadian clock of the pancreas is required for normal insulin release and glucose homeostasis in mice. Diabetologia 54: 120-124.

Saini C, Morf J, Stratmann M, Gos P, Schibler U. 2012. Simulated body temperature rhythms reveal the phase-shifting behavior and plasticity of mammalian circadian oscillators. Genes Dev 26: 567-580.

Saini C, Liani A, Curie T, Gos P, Kreppel F, Emmenegger Y, Bonacina L, Wolf JP, Poget YA, Franken P, et al. 2013. Realtime recording of circadian liver gene expression in freely moving mice reveals the phase-setting behavior of hepatocyte clocks. Genes Dev 27: 1526-1536.

Schaeren-Wiemers N, Andre E, Kapfhammer JP, Becker-André M. 1997. The expression pattern of the orphan nuclear receptor ROR $\beta$ in the developing and adult rat nervous system suggests a role in the processing of sensory information and in circadian rhythm. Eur J Neurosci 9: 2687-2701.

Sharma VK, Chandrashekaran MK. 1998. Age-dependent modulation of circadian parameters in the field mouse Mus booduga. J Exp Zool 280: 321-326.

Tosches MA, Bucher D, Vopalensky P, Arendt D. 2014. Melatonin signaling controls circadian swimming behavior in marine zooplankton. Cell 159: 46-57.

Yamajuku D, Inagaki T, Haruma T, Okubo S, Kataoka Y, Kobayashi S, Ikegami K, Laurent T, Kojima T, Noutomi K, et al. 2012. Real-time monitoring in three-dimensional hepatocytes reveals that insulin acts as a synchronizer for liver clock. Sci Rep 2: 439.

Ye R, Selby CP, Chiou Y-Y, Ozkan-Dagliyan I, Gaddameedhi S, Sancar A. 2014. Dual modes of CLOCK:BMAL1 inhibition mediated by Cryptochrome and Period proteins in the mammalian circadian clock. Genes Dev 28: 1989-1998.

Yoo SH, Yamazaki S, Lowrey PL, Shimomura K, Ko CH, Buhr ED, Siepka SM, Hong HK, Oh WJ, Yoo OJ, et al. 2004. PERIOD2::LUCIFERASE real-time reporting of circadian dynamics reveals persistent circadian oscillations in mouse peripheral tissues. Proc Natl Acad Sci 101: 53395346.

Zmrzljak UP, Rozman D. 2012. Circadian regulation of the hepatic endobiotic and xenobitoic detoxification pathways: The time matters. Chem Res Toxicol 25: 811-824. 


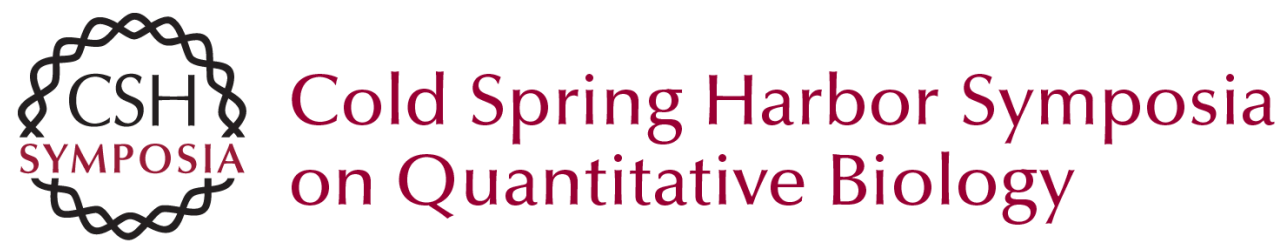

\section{Clock-Talk: Interactions between Central and Peripheral Circadian Oscillators in Mammals}

Ueli Schibler, Ivana Gotic, Camille Saini, et al.

Cold Spring Harb Symp Quant Biol 2015 80: 223-232 originally published online December 18, 2015

Access the most recent version at doi:10.1101/sqb.2015.80.027490

References This article cites 52 articles, 17 of which can be accessed free at: http://symposium.cshlp.org/content/80/223.full.html\#ref-list-1

\section{License}

Email Alerting Receive free email alerts when new articles cite this article - sign up in Service the box at the top right corner of the article or click here. 